COMMUNICATIONS IN

ANALYSIS AND GEOMETRY

Volume 11, Number 4, 697-719, 2003

\title{
Infinitesimal Bendings of Homogeneous Surfaces with Nonnegative Curvature
}

\author{
Abdelhamid Meziani
}

\section{Introduction.}

This paper deals with infinitesimal bendings of a surface $S$ in a neighborhood of a point $p \in S$. More precisely, consider a surface $S$ embedded in $\mathbf{R}^{3}$ and given by parametric equation

$$
R(u, v)=(x(u, v), y(u, v), z(u, v)) \in \mathbf{R}^{3},
$$

with $(u, v) \in \mathbf{R}^{2}$ and $p=R(0,0)=0$. An infinitesimal bending of $S$ is a deformation $S_{t}$, with $-\delta<t<\delta$, given by an embedding

$$
R_{t}(u, v)=R(u, v)+t U(u, v),
$$

such that the first fundamental forms of $S_{t}$ and $S$ satisfy

$$
d s_{t}^{2}=d s^{2}+O\left(t^{2}\right)
$$

The main question is whether a given surface $S$ admits nontrivial infinitesimal bendings in a neighborhood of $p$. By nontrivial infinitesimal bendings we mean those bendings that are not induced by the rigid motions of the ambient space $\mathbf{R}^{3}$. The study of this question and related problems is very old and goes back at least to Darboux ([D]). Local and global aspects of such problems, as well as the physical applications (elasticity of thin shells for example), have been studied in $[\mathrm{A}],[\mathrm{BG}],[\mathrm{C}],[\mathrm{D}],[\mathrm{E} 1],[\mathrm{E} 2],[\mathrm{EU}],[\mathrm{K}]$, $[\mathrm{P}],[\mathrm{S} 1],[\mathrm{S} 2],[\mathrm{S} 3],[\mathrm{S}],[\mathrm{U} 1],[\mathrm{U} 2],[\mathrm{V}]$.

Let $K(u, v)$ be the gaussian curvature of $S$. When $K(0) \neq 0$, the infinitesimal bendings of $S$ are well understood (see [D] or [V]). The case $K(0)=0$ leads to equations with singularities. In 1948, Efimov [E3] proved that the surface $\left(u, v, u^{9}+\lambda u^{7} v^{2}+v^{9}\right)$, with $\lambda \in \mathbf{R}$ a transcendental number, is rigid under real analytic bendings. Later on, he proved that most real analytic surfaces are rigid under real analytic bendings. 
For surfaces with $K(0)=0$, the only case that is well understood is the case of rotation surfaces $\left(u, v,\left(u^{2}+v^{2}\right)^{m}\right)$ and their perturbations: surfaces of the form

$$
\left(u, v,\left(u^{2}+v^{2}\right)^{m} f(u, v)\right)
$$

with $f(0) \neq 0$ (see [U1] and [U2]).

In this paper we consider surfaces $S$ of the form

$$
R(u, v)=\left(u, v, F_{m}(u, v)\right)
$$

with $F_{m}(u, v) \geq 0$ a homogeneous function of order $m$. We assume that the surface is located on one side of its tangent plane at 0 ; that it has nonnegative gaussian curvature $(K \geq 0)$; and that the curvature is almost everywhere positive (the homogeneity of $F$ implies that the set of points where $K=0$ is a union of curves through 0 ). We prove (section 3 ) that the surface $S$ admits nontrivial infinitesimal bendings. In section 5 , we consider the case when $S$ has positive curvature except at 0 . In this case we give a complete description of the space of infinitesimal bendings. We approach the problem by studying the induced Hamiltonian systems and using eigenfunctions expansion. A method used recently by the author to study singular Cauchy-Riemann equations [M2].

\section{Equations for the bending field.}

In this section, we recall the definitions and the systems of differential equations for the field of infinitesimal bending.

Let $S$ be a surface of class $C^{l}$, with $l>2$, in $\mathbf{R}^{3}$. We can choose coordinates $(x, y, z)$ in $\mathbf{R}^{3}$ so that $0 \in S, S$ is given near 0 by the graph of a function $z=z(x, y)$, and $S$ is tangent at 0 to the $x y$-plane. That is,

$$
S=\left\{R(x, y)=(x, y, z(x, y)) \in \mathbf{R}^{3}:(x, y) \in D_{\epsilon}\right\},
$$

where $D_{\epsilon}$ is the disc with center at 0 and radius $\epsilon, z \in C^{l}\left(D_{\epsilon}\right), z(0)=0$, and $z_{x}(0)=z_{y}(0)=0$. An infinitesimal bending of $S$ of class $C^{l^{\prime}}$ is a deformation $S_{t}$ given by

$$
S_{t}=\left\{R_{t}(x, y)=R(x, y)+t U(x, y) \in \mathbf{R}^{3}:(x, y) \in D_{\epsilon}\right\},
$$

with $-\delta<t<\delta(\delta>0)$ and $U \in C^{l^{\prime}}\left(D_{\epsilon} ; \mathbf{R}^{3}\right)$, and such that the first fundamental forms satisfy

$$
d R_{t}^{2}=d R^{2}+O\left(t^{2}\right) \quad-\delta<t<\delta .
$$


Note that since

$$
d R_{t}^{2}=d R^{2}+2 t d R \cdot d U+t^{2} d U^{2}
$$

then in order for (2.3) to hold, it is necessary that the field of infinitesimal bending $U$ satisfies the equation

$$
d R \cdot d U=0 \quad \text { in } D_{\epsilon} .
$$

Hence, under an infinitesimal bending, the curves of $S$ undergo only stretching. Also, if $\Sigma_{t}$ is an isometric deformation of $S$ given by the position vector $\tilde{R}_{t}(x, y)$ (with $\tilde{R}_{0}=R$ ), then the linear approximation $R+t \frac{d \tilde{R}_{0}}{d t}$ is an infinitesimal bending of $S$.

If $A, B \in \mathbf{R}^{3}$, then

$$
R(x, y)+t(A \times R(x, y)+B)
$$

where $\times$ denotes the vector product in $\mathbf{R}^{3}$, is an infinitesimal bending of $S$. These infinitesimal bendings are said to be trivial. A surface $S$ is said to be rigid if its only infinitesimal bendings are the trivial ones.

Let

$$
U(x, y)=(\xi(x, y), \eta(x, y), \zeta(x, y))
$$

be a field of infinitesimal bending of $S$, where $\xi, \eta$, and $\zeta$ are in $C^{l^{\prime}}\left(D_{\epsilon} ; \mathbf{R}\right)$. In terms of these functions, equation (2.5) takes the form

$$
\begin{aligned}
\xi_{x}+z_{x} \zeta_{x} & =0 \\
\eta_{y}+z_{y} \zeta_{y} & =0 \\
\xi_{y}+\eta_{x}+z_{x} \zeta_{y}+z_{y} \zeta_{x} & =0
\end{aligned}
$$

The elimination of $\xi$ and $\eta$ in the system (2.8) leads to the second order pde for $\zeta($ see $[\mathrm{V}])$

$$
z_{y y} \zeta_{x x}-2 z_{x y} \zeta_{x y}+z_{x x} \zeta_{y y}=0
$$

For every solution $\zeta$ of (2.9), the system (2.8) can be solved by quadrature for $\xi$ and $\eta$ to produce the field $U$. The system (2.8) can also be reduced to a first order $2 \times 2$ system as follows. Let

$$
f=\xi+z_{x} \zeta \text { and } g=\eta+z_{y} \zeta
$$

It follows at once from (2.8) that

$$
f_{x}=z_{x x} \zeta, \quad g_{y}=z_{y y} \zeta, \quad \text { and } \quad f_{y}+g_{x}=2 z_{x y} \zeta
$$


The elimination of $\zeta$ leads to the system

$$
\begin{aligned}
& 2 z_{x y} f_{x}-z_{x x}\left(f_{y}+g_{x}\right)=0 \\
& 2 z_{x y} g_{y}-z_{y y}\left(f_{y}+g_{x}\right)=0
\end{aligned}
$$

A solution $(f, g)$ of $(2.12)$ corresponds to a solution $(\xi, \eta, \zeta)$ of $(2.8)$. Thus finding a bending field $U$ is equivalent to solving either of the equations (2.8), $(2.9)$, or $(2.12)$.

Let $K(x, y)$ be the Gaussian curvature of $S$. From now on we will assume that $K(x, y) \geq 0$. Recall that

$$
K(x, y)=\frac{z_{x x} z_{y y}-z_{x y}^{2}}{\left(1+z_{x}^{2}+z_{y}^{2}\right)^{2}} .
$$

Without loss of generality, we assume that

$$
z_{x x}(x, y) \geq 0 \quad z_{y y}(x, y) \geq 0 \quad \text { for } \quad(x, y) \neq(0,0) \cdot(2.14)
$$

Remark 2.1 Let

$$
\vartheta=\frac{-z_{x y}+i \sqrt{z_{x x} z_{y y}-z_{x y}^{2}}}{z_{y y}}
$$

be the (complex) asymptotic direction of $S$ and let

$$
w=f+\vartheta g,
$$

where $f$ and $g$ are given in (2.11). A direct calculation shows that the system (2.12) is equivalent to the equation

$$
(\vartheta-\bar{\vartheta}) L w=L \vartheta(w-\bar{w})
$$

where $L$ is the complex vector field

$$
L=\frac{\partial}{\partial x}+\vartheta \frac{\partial}{\partial y} .
$$

When the curvature $K$ vanishes only at 0 and is positive elsewhere, then equation (2.17) can be considerably simplified by using polar coordinates and a normalization result (see $[\mathrm{M}]$ ). It is then equivalent to an equation of the form

$$
\left(\frac{\partial}{\partial \theta}+i r c(\theta) \frac{\partial}{\partial r}\right) w=A(r, \theta)(w-\bar{w})
$$


in a neighborhood of the circle $S^{1} \times\{0\} \subset S^{1} \times \mathbf{R}$, where $c(\theta)$ is a function depending on $\theta$ alone and satisfies $\operatorname{Rec}(\theta) \neq 0$. The vector field $\frac{\partial}{\partial \theta}+\operatorname{irc}(\theta) \frac{\partial}{\partial r}$ is of infinite type along the circle $S^{1} \times\{0\}$ (see [T] or [M1]). For surfaces of nonnegative curvature, the study of infinitesimal bendings is therefore closely connected to solvability of complex vector fields.

\section{Existence of infinitesimal bendings.}

In this section we prove that the graph of a homogeneous function with a positive curvature almost everywhere has nontrivial infinitesimal bendings. More precisely, we have the following result.

Theorem 3.1 Let $z(x, y)$ be a homogeneous function of order $m$ and of class $C^{l}$, with $3 \leq l \leq \infty$. Let $S$ be the graph of $z$. Suppose that $z(x, y)>0$ for $(x, y) \neq 0$ and that the Gaussian curvature $K(x, y)$ is almost everywhere positive. Then, for $l<\infty$, there exist $U \in C^{l-2}\left(\mathbf{R}^{2} ; \mathbf{R}^{3}\right)$ such that

$$
R_{t}(x, y)=(x, y, z(x, y))+t U(x, y)
$$

is a nontrivial infinitesimal bending of $S$. When $l=\infty$, then for every $N \in \mathbf{Z}^{+}$, the surface $S$ admits a nontrivial bending field $U \in C^{N}\left(\mathbf{R}^{2} ; \mathbf{R}^{3}\right)$.

The remainder of this section is devoted to the proof of the theorem. The idea is to solve system (2.12) using methods of Hamiltonian differential equations. We start by rewriting the system using polar coordinates

$$
x=r \cos \theta \quad \text { and } \quad y=r \sin \theta .
$$

The homogeneous function $z$ takes the form

$$
z=r^{m} P(\theta)
$$

where $P \in C^{l}(\mathbf{R})$ is $2 \pi$-periodic and

$$
P(\theta)>0 \quad \forall \theta \in[0,2 \pi] .
$$

The nonnegativity of the curvature is expressed in terms of the function $P$ as

$$
m^{2} P(\theta)^{2}+m P(\theta) P^{\prime \prime}(\theta)-(m-1) P^{\prime}(\theta)^{2} \geq 0 .
$$


Furthermore, the above inequality is strict for almost every $\theta$. To rewrite the system (2.12) in polar coordinates, we need the partial derivatives of $z$ in terms of $r$ and $\theta$ :

$$
\begin{aligned}
& z_{x x}=r^{m-2}\left[\left(m(m-1) \cos ^{2} \theta+m \sin ^{2} \theta\right) P-2(m-1) \cos \theta \sin \theta P^{\prime}+\sin ^{2} \theta P^{\prime \prime}\right] \\
& z_{x y}=r^{m-2}\left[m(m-2) \cos \theta \sin \theta P-(m-1)\left(\cos ^{2} \theta-\sin ^{2} \theta\right) P^{\prime}-\sin \theta \cos \theta P^{\prime \prime}\right] \\
& z_{y y}=r^{m-2}\left[\left(m(m-1) \sin ^{2} \theta+m \cos ^{2} \theta\right) P+2(m-1) \cos \theta \sin \theta P^{\prime}+\cos ^{2} \theta P^{\prime \prime}\right]
\end{aligned}
$$

Let

$$
V=\left(\begin{array}{l}
f \\
g
\end{array}\right)
$$

where $f$ and $g$ are given by (2.10). The system (2.12) is then equivalent to the system

$$
\frac{1}{r} C V_{\theta}=D V_{r}
$$

where

$$
\begin{gathered}
C=\left(\begin{array}{cc}
z_{y y} \sin \theta & z_{x x} \cos \theta \\
-z_{y y} \cos \theta & 2 z_{x y} \cos \theta+z_{y y} \sin \theta
\end{array}\right), \\
D=\left(\begin{array}{cc}
z_{y y} \cos \theta & -z_{x x} \sin \theta \\
z_{y y} \sin \theta & -2 z_{x y} \sin \theta+z_{y y} \cos \theta
\end{array}\right) .
\end{gathered}
$$

Equation (3.8) can be written as

$$
\frac{1}{r} V_{\theta}=M V_{r}
$$

where

$$
\begin{aligned}
M & =\frac{1}{r^{m-2} m(m-1) P(\theta)}\left(\begin{array}{cc}
a & b \\
c & d
\end{array}\right) \\
a & =2 z_{x y} \cos ^{2} \theta+\left(z_{y y}-z_{x x}\right) \cos \theta \sin \theta \\
b & =-z_{x x} \\
c & =z_{y y} \\
d & =-2 z_{x y} \sin ^{2} \theta+\left(z_{y y}-z_{x x}\right) \cos \theta \sin \theta
\end{aligned}
$$

Note that $M=M(\theta)$ is independent on the radius $r$. By using (3.11) and expressions (3.6), it is verified that the trace of $M$ is

$$
\operatorname{Tr}(M)=\frac{2 P^{\prime}(\theta)}{m P(\theta)} .
$$

It will be more convenient to us to have a system in which the trace is 0 . To achieve this situation, we make the change of variables where the new radius is

$$
\rho=r P(\theta)^{\frac{1}{m}} .
$$


With respect to the coordinates $(\rho, \theta)$, the system (3.10) becomes

$$
\frac{1}{\rho} V_{\theta}=\left(M-\frac{P^{\prime}}{m P} I\right) V_{\rho}=A(\theta) V_{\rho}
$$

Now the matrix $A$ has trace zero and is given by

$$
A(\theta)=\frac{1}{m(m-1) r^{m-2} P}\left(\begin{array}{cc}
z_{x y} & -z_{x x} \\
z_{y y} & -z_{x y}
\end{array}\right) .
$$

We will seek solutions of (3.14) in the form

$$
V(\rho, \theta)=\rho^{\lambda} X(\theta),
$$

with $\lambda \in \mathbf{R}$ and $X(\theta)$ a $2 \pi$-periodic function of $\theta$. A function $V$, in (3.16), solves (3.14) if and only if the function $X$ satisfies the ode

$$
X^{\prime}(\theta)=\lambda A(\theta) X(\theta)
$$

We rewrite (3.17) in the form

$$
J X^{\prime}(\theta)=\lambda H(\theta) X(\theta)
$$

with

$$
J=\left(\begin{array}{cc}
0 & -1 \\
1 & 0
\end{array}\right), \quad H=J A=\frac{1}{m(m-1) r^{m-2} P}\left(\begin{array}{cc}
z_{y y} & -z_{x y} \\
-z_{x y} & z_{x x}
\end{array}\right) .
$$

The next proposition gives the spectrum of equation (3.18).

Proposition 3.1 There exists a sequence

$$
\cdots<\lambda_{-2}^{-} \leq \lambda_{-2}^{+}<\lambda_{-1}^{-} \leq \lambda_{-1}^{+}<\lambda_{0}=0<\lambda_{1}^{-} \leq \lambda_{1}^{+}<\lambda_{2}^{-} \leq \lambda_{2}^{+}<\cdots
$$

with

$$
\lim _{j \rightarrow \pm \infty} \lambda_{j}^{ \pm}= \pm \infty
$$

such that equation

$$
J X^{\prime}=\lambda H X
$$

has a nontrivial periodic solution if and only if $\lambda=\lambda_{j}^{ \pm}$for some $j \in \mathbf{Z}$. Furthermore, the fundamental matrix of $(3.21)_{\lambda_{j}^{+}}$is $2 \pi$-periodic if and only if $\lambda_{j}^{+}=\lambda_{j}^{-}$. 
Proof. It follows from the hypotheses $\left(z(x, y)>0\right.$ for $(x, y) \neq 0, z \in C^{l}$, with $l \geq 3$, and $K(x, y)>0$ almost everywhere) that the eigenvalues $h_{-}$and $h_{+}$of $H$ depend continuously on $\theta$ and they satisfy

$$
0 \leq h_{-}(\theta) \leq h_{+}(\theta) \quad \forall \theta \in[0,2 \pi] .
$$

and that $h_{-}(\theta)>0$ for almost every $\theta$. Hence,

$$
\int_{0}^{2 \pi} h_{-}(\theta) d \theta>0 .
$$

This is a sufficient condition for equation (3.18) to have spectrum as in the proposition (see [YS] Chapter VIII page 762) $\triangle$

Remark 3.1 For every $\lambda \in\left(\lambda_{j}^{-}, \lambda_{j}^{+}\right)$, equation $(3.21)_{\lambda}$ is unstable. For every $\lambda \in\left(\lambda_{j}^{+}, \lambda_{j+1}^{-}\right)$, equation $(3.21)_{\lambda}$ is stable. All solutions of $(3.21)_{\lambda_{j}^{+}}$ are $2 \pi$-periodic if and only if $\lambda_{j}^{-}=\lambda_{j}^{+}$(see [YS] page 761 ).

Now we will construct a field of infinitesimal bending for the surface $S$. For $j \in \mathbf{Z}$, let

$$
\varphi_{j}^{ \pm}(\theta)=\left(\begin{array}{c}
p_{j}^{ \pm}(\theta) \\
q_{j}^{ \pm}(\theta)
\end{array}\right) \in C^{l-1}\left(\mathbf{R} ; \mathbf{R}^{2}\right)
$$

be an eigenfunction of (3.18) corresponding to the eigenvalue $\lambda_{j}^{ \pm}$. The functions

$$
f(r, \theta)=r^{\lambda_{j}^{ \pm}} P(\theta)^{\frac{\lambda_{j}^{ \pm}}{m}} p_{j}^{ \pm}(\theta), \quad g(r, \theta)=r^{\lambda_{j}^{ \pm}} P(\theta)^{\frac{\lambda_{j}^{ \pm}}{m}} q_{j}^{ \pm}(\theta)
$$

solve the system (2.12).

It follows from (3.4), (3.5), and expressions (3.6) that

$$
\frac{z_{x x}+z_{y y}}{r^{m-2}}=m^{2} P(\theta)+P^{\prime \prime}(\theta)>0 \quad \forall \theta .
$$

Let

$$
\zeta(r, \theta)=\frac{f_{x}+g_{y}}{z_{x x}+z_{y y}}=r^{\lambda_{j}^{ \pm}-m+1} \gamma_{j}^{ \pm}(\theta),
$$

where $\gamma_{j}^{ \pm} \in C^{l-2}(\mathbf{R})$ is $2 \pi$-periodic. Then the functions $\xi$ and $\eta$ are

$$
\begin{aligned}
& \xi=f-z_{x} \zeta=r^{\lambda_{j}^{ \pm}} \alpha_{j}^{ \pm}(\theta) \\
& \eta=g-z_{y} \zeta=r^{\lambda_{j}^{ \pm}} \beta_{j}^{ \pm}(\theta)
\end{aligned}
$$


where $\alpha_{j}^{ \pm}$and $\beta_{j}^{ \pm}$are $2 \pi$-periodic and in $C^{l-2}(\mathbf{R})$. The field

$$
U_{j}^{ \pm}(r, \theta)=\left(r^{\lambda_{j}^{ \pm}} \alpha_{j}^{ \pm}(\theta), r^{\lambda_{j}^{ \pm}} \beta_{j}^{ \pm}(\theta), r^{\lambda_{j}^{ \pm}-m+1} \gamma_{j}^{ \pm}(\theta)\right)
$$

is an infinitesimal bending of the surface $S$. It is clear that if $\lambda_{j}^{ \pm}$is large enough, then $U_{j}$ is in the class $C^{l-2}$. Furthermore, when $l=\infty$, the eigenfunctions $\varphi_{j}^{ \pm} \in C^{\infty}$ and so are the functions $\alpha_{j}^{ \pm}, \beta_{j}^{ \pm}$and $\gamma_{j}^{ \pm}$. The field $U_{j}^{ \pm}$is then $C^{\infty}$ away from 0 and for any given $N \in \mathbf{Z}^{+}$, we can take $j$ large enough so that $U_{j}^{ \pm}$vanishes to high order at 0 in such a way that it is of class $C^{N}$ at 0 . This completes the proof of the theorem $\triangle$

\section{Periodicity of the fundamental matrices.}

In this section, we prove that the fundamental matrices of $(3.21)_{\lambda}$ are periodic for each $\lambda$ in the spectrum. We first prove the result when $P(\theta)$ is a trigonometric polynomial.

Lemma 4.1 Let $z$ be as in (3.3) with $P(\theta)$ a trigonometric polynomial satisfying (3.4) and (3.5) and let $J$ and $H$ be the matrices defined in (3.19). Let

$$
\Sigma=\left\{\lambda_{j}^{ \pm} ; j \in \mathbf{Z}\right\}
$$

be the spectrum of the equation

$$
J X^{\prime}(\theta)=\lambda H(\theta) X(\theta) .
$$

Then for every $\lambda_{j}^{ \pm} \in \Sigma$, the fundamental matrix of $(4.2)_{\lambda_{j}^{ \pm}}$is $2 \pi$-periodic.

Proof. It follows from Floquet theory (see [YS] Chapter VIII page 617) that the fundamental matrix of $(4.2)_{\lambda}$ has the form

$$
W_{\lambda}(\theta)=F_{\lambda}(\theta) \mathrm{e}^{\theta K_{\lambda}}
$$

where $F_{\lambda}$ is a $2 \pi$ periodic $2 \times 2$ matrix and $K_{\lambda}$ is a constant matrix satisfying

$$
\operatorname{Tr}\left(K_{\lambda}\right)=0
$$

Note that since here $H(\theta)$ is real analytic, then the solutions of $(4.2)_{\lambda}$ are real analytic. The monodromy matrix of $(4.2)_{\lambda}$ is

$$
B_{\lambda}=\mathrm{e}^{2 \pi K_{\lambda}}
$$


and has determinant equal to 1 . The characteristic exponents have the form

$$
\rho_{1}(\lambda)=\mathrm{e}^{\mu(\lambda)} \quad \text { and } \quad \rho_{2}(\lambda)=\frac{1}{\rho_{1}(\lambda}=\mathrm{e}^{-\mu(\lambda)},
$$

where $\mu(\lambda)$ and $-\mu(\lambda)$ are the eigenvalues of $K_{\lambda}$ and $\mu(\lambda) \in \mathbf{R}$ or $\mu(\lambda) \in i \mathbf{R}$. Furthermore, $\mu$ depends continuously on $\lambda$ and

$$
\mu(\lambda)=0 \quad \text { if and only if } \quad \lambda=\lambda_{j} \in \Sigma .
$$

It follows that

$$
K_{\lambda_{j}}=0 \quad \text { or } \quad K_{\lambda_{j}} \sim\left(\begin{array}{cc}
0 & 1 \\
0 & 0
\end{array}\right), \quad \lambda_{j} \in \Sigma,
$$

where $\sim$ stands for similarity of matrices. To prove the lemma, we need to show that $K_{\lambda_{j}}=0$.

To show the vanishing of $K_{\lambda_{j}}$, we will make use of the second order pde (2.9). We will show that the corresponding pde has two independent solutions. We rewrite (2.9) in polar coordinates (using $z=r^{m} P(\theta)$ ):

$$
\begin{aligned}
\left(m P+P^{\prime \prime}\right) \zeta_{r r}-\frac{2(m-1)}{r} P^{\prime} \zeta_{r \theta} & +\frac{m(m-1)}{r^{2}} P \zeta_{\theta \theta}+ \\
& \frac{m(m-1)}{r} P \zeta_{r}+\frac{2(m-1)}{r^{2}} P^{\prime} \zeta_{\theta}=0 .
\end{aligned}
$$

Equation (4.9) has a solution $\zeta$ of the form

$$
\zeta=r^{\sigma} \gamma(\theta)
$$

if and only if the $2 \pi$ periodic function $\gamma$ solves the Sturmian problem

$$
\begin{gathered}
m(m-1) P \gamma^{\prime \prime}-2(m-1)(\sigma-1) P^{\prime} \gamma^{\prime}+\sigma\left[(\sigma-1) P^{\prime \prime}+(m+\sigma-2)\right] \gamma=0 \\
\gamma(0)=\gamma(2 \pi)
\end{gathered}
$$

We know, thanks to proposition 3.1 and relations (2.9) and (2.12) (see also(3.27)), that the spectrum of problem (4.11) consists of

$$
\sigma_{j}^{ \pm}=\lambda_{j}^{ \pm}-(m-1) \quad \text { with } \quad \lambda_{j}^{ \pm} \in \Sigma .
$$

To prove that (4.11) has two independent solutions, we extend it to the Riemann sphere $\overline{\mathbf{C}}$ and consider it as a Fuchsian equation.

We write the positive trigonometric polynomial $P$ as

$$
P(\theta)=a_{0}+\sum_{j=1}^{s} a_{j} \mathrm{e}^{i j \theta}+\sum_{j=1}^{s} \overline{a_{j}} \mathrm{e}^{-i j \theta}
$$


and extend it as a rational function $R(w)$ on $\overline{\mathbf{C}}$ as

$$
R(w)=a_{0}+\sum_{j=1}^{s} a_{j} w^{j}+\sum_{j=1}^{s} \overline{a_{j}} \frac{1}{w^{j}} .
$$

Let $f(w)$ be the holomorphic extension of the function $\gamma(\theta)$ considered a real analytic on the unit circle in $\overline{\mathbf{C}}$. Since

$$
P^{\prime}(\theta)=R^{\prime}\left(\mathrm{e}^{i \theta}\right) i \mathrm{e}^{i \theta}, \quad P^{\prime \prime}(\theta)=-R^{\prime \prime}\left(\mathrm{e}^{i \theta}\right) \mathrm{e}^{2 i \theta}-R^{\prime}\left(\mathrm{e}^{i \theta}\right) \mathrm{e}^{i \theta}
$$

and similar relations hold between the derivatives of $f$ and $\gamma$, then a calculation shows that $\gamma(\theta)$ solves (4.11) if and only if $f(w)$ solves the differential equation

$$
f^{\prime \prime}(w)+A_{\sigma}(w) f^{\prime}(w)+B_{\sigma}(w) f(w)=0,
$$

with

$$
\begin{aligned}
& A_{\sigma}(w)=\frac{1}{w}-\frac{2(\sigma-1)}{m} \frac{R^{\prime}(w)}{R(w)} \\
& B_{\sigma}(w)=\sigma\left(\frac{\sigma-1}{m(m-1)} \frac{R^{\prime \prime}(w)}{R(w)}+\frac{\sigma-1}{m(m-1)} \frac{1}{w} \frac{R^{\prime}(w)}{R(w)}-\frac{m+\sigma-2}{m-1} \frac{1}{w^{2}}\right)
\end{aligned}
$$

We will prove that for each $\sigma=\sigma_{j}^{ \pm}$(given by (4.12)), the Fuchsian equation $(4.16)_{\sigma_{j}^{ \pm}}$has two independent solutions that are holomorphic in a neighborhood of the unit circle. For this, we show that the monodromy matrix corresponding to the unit circle is the identity. We know that for $\sigma \neq \sigma_{j}^{ \pm}$, the monodromy matrix of $(4.16)_{\sigma}$ has eigenvalues

$$
\mathrm{e}^{\mu(\sigma)} \text { and } \mathrm{e}^{-\mu(\sigma)}
$$

with $\mu$ satisfying (4.7). Let $f_{j}(w)$ be a nonzero solution of $(4.16)_{\sigma_{j}^{ \pm}}$such that $f_{j}$ is holomorphic in a neighborhood of the unit circle. For $\delta>0$, consider the annulus

$$
O_{\delta}=\{w \in \mathbf{C}: 1-\delta<|z|<1+\delta\} .
$$

Choose $\delta$ small enough so that

$$
R(w) \neq 0 \quad \forall w \in O_{\delta} \text { and } f_{j}(w) \neq 0 \quad \forall w \in O_{\delta} \text { with }|w| \neq 1 .
$$

Note that such a choice of $\delta$ is possible since $R\left(\mathrm{e}^{i \theta}\right)=P(\theta)>0$ and $f_{j}$, holomorphic near the circle, has isolated zeros. Since the coefficient $A_{\sigma}$ and $B_{\sigma}$ of (4.16) depend analytically on the parameter $\sigma$, then we can find a 
(multivalued) solution $U_{\sigma}(w)$, that depends analytically on $\sigma$, and such that $U_{\sigma_{j}^{ \pm}}=f_{j}$. For $\sigma \neq \sigma_{j}^{ \pm}$and $\left|\sigma-\sigma_{j}^{ \pm}\right|<\tau(\tau$ small), equation (4.16) $\sigma$ has two independent solutions of the form

$$
F_{\sigma}^{1}(w)=w^{\alpha(\sigma)} G_{\sigma}^{1}(w) \text { and } \quad F_{\sigma}^{2}(w)=w^{-\alpha(\sigma)} G_{\sigma}^{2}(w)
$$

with $\mu(\sigma)=2 \pi i \alpha(\sigma)$ and $G_{\sigma}^{1}, G_{\sigma}^{2}$ holomorphic in the annulus $O_{\delta}$. We can further assume that the functions $G_{\sigma}^{1}$ and $G_{\sigma}^{2}$ are uniformly bounded in $O_{\delta}$ (replace $G_{\sigma}^{1}$ and $G_{\sigma}^{1}$ by their multiples, if necessary). This implies that these families of holomorphic functions have convergent subsequences as $\sigma$ approaches $\sigma_{j}$. We can therefore assume that

$$
G_{\sigma_{j}^{ \pm}}^{k}(w)=\lim _{\sigma \rightarrow \sigma_{j}^{ \pm}} G_{\sigma}^{k}(w), \quad k=1,2
$$

are holomorphic functions in $O_{\delta}$. Since $U_{\sigma}$ is a linear combination of $F_{\sigma}^{1}$ and $F_{\sigma}^{2}$, then it follows from (4.7) and (4.22) that $f_{j}$ is a linear combination of the functions $G_{\sigma_{j}^{ \pm}}^{1}$ and $G_{\sigma_{j}^{ \pm}}^{2}$. The next step is to prove that $G_{\sigma_{j}^{ \pm}}^{1}$ and $G_{\sigma_{j}^{ \pm}}^{2}$ are independent solutions of $(4.16)_{\sigma_{j}^{ \pm}}$.

Since a linear combination of $G_{\sigma_{j}^{ \pm}}^{1}$ and $G_{\sigma_{j}^{ \pm}}^{2}$ gives the nonzero function $f_{j}$, then we can assume that $G_{\sigma_{j}^{ \pm}}^{1}(1) \neq 0$. Let $C$ be a loop based at 1 , contained in the annulus $O_{\delta}$, homotopic to the unit circle in $O_{\delta}$, and such that $G_{\sigma_{j}^{ \pm}}^{1}(w) \neq 0$ for all points $w \in C$. Let $N(C)$ be a tubular neighborhood of $C$, such that $N(C) \subset O_{\delta}$ and

$$
G_{\sigma}^{1}(w) \neq 0 \quad \forall w \in N(C), \quad \forall \sigma,\left|\sigma-\sigma_{j}\right|<\tau .
$$

Starting with the solution $F_{\sigma}^{1}(w)$ of $(4.16)_{\sigma}$, we construct a second solution $\hat{F}_{\sigma}^{1}(w)$ by the method of variation of parameter. We find

$$
\hat{F}_{\sigma}^{1}(w)=F_{\sigma}^{1}(w) I_{\sigma}^{1}(w), \quad w \in N(C)
$$

with

$$
I_{\sigma}^{1}(w)=\int_{\Gamma(1, w)} \frac{1}{s} \frac{R(s)^{2(\sigma-1) / m}}{F_{\sigma}^{1}(s)} d s
$$

where $\Gamma(1, w)$ is a curve in $N(C)$ connecting 1 to $w$. Of course $I_{\sigma}^{1}(w)$ could be multivalued and a holomorphic branch is well defined in any simply connected domain in $N(C)$ containing 1 . The new solution $\hat{F}_{\sigma}^{1}(w)$ is also a 
linear combination of $F_{\sigma}^{1}$ and $F_{\sigma}^{2}$. Hence there exist constants $C_{\sigma}^{1}$ and $C_{\sigma}^{2}$ such that

$\hat{F}_{\sigma}^{1}(w)=C_{\sigma}^{1} w^{\alpha(\sigma)} G_{\sigma}^{1}(w)+C_{\sigma}^{2} w^{-\alpha(\sigma)} G_{\sigma}^{2}(w) \quad \forall w \in N(C), \quad \forall \sigma:\left|\sigma-\sigma_{j}\right|<\tau$.

It follows from (4.24) and (4.26) that

$$
I_{\sigma}^{1}(w)=C_{\sigma}^{1}+C_{\sigma}^{2} w^{-2 \alpha(\sigma)} \frac{G_{\sigma}^{2}(w)}{G_{\sigma}^{1}(w)} .
$$

Hence,

$$
I_{\sigma_{j}^{ \pm}}^{1}(w)=\lim _{\sigma \rightarrow \sigma_{j}^{ \pm}} I_{\sigma}^{1}(w)=C_{\sigma_{j}^{ \pm}}^{1}+C_{\sigma_{j}^{ \pm}}^{2} \frac{G_{\sigma_{j}^{ \pm}}^{2}(w)}{G_{\sigma_{j}^{ \pm}}^{1}(w)}
$$

is holomorphic in $N(C)$. Therefore

$$
\hat{F}_{\sigma_{j}^{ \pm}}^{1}(w)=F_{\sigma_{j}^{ \pm}}^{1}(w) I_{\sigma_{j}^{ \pm}}^{1}(w)
$$

is a second (independent) holomorphic solution of $(4.16)_{\sigma_{j}^{ \pm}}$in $N(C)$. Hence the monodromy matrix of $(4.16)_{\sigma_{j}^{ \pm}}$corresponding to the loop $C$ is the identity. Since the matrix depends only on the homotopy class of the loop (see [Y] for example), and since $C$ and the unit circle are homotopic in $O_{\delta}$, then the monodromy matrix of $(4.16)_{\sigma_{j}^{ \pm}}$corresponding to the unit circle is the identity. This completes the proof of the lemma $\triangle$

This lemma together with Remark 3.1 give the following consequence

Corollary 4.1 Under the hypotheses of lemma 4.1, equation $(4.2)_{\lambda}$ is stable for every $\lambda \in \mathbf{R}$.

To prove the result when $P$ is not real analytic, we will use a property of the rotation function for solutions of a system

$$
J \dot{Y}(\theta)=A(\theta) Y(\theta)
$$

where $A$ is a 2 by 2 symmetric matrix with periodic coefficients. Let

$$
Y(\theta, \nu)=\left(\begin{array}{l}
y_{1}(\theta, \nu) \\
y_{2}(\theta, \nu)
\end{array}\right)
$$


be the solution of (4.31) such that $y_{1}(0, \nu)=\cos \nu$ and $y_{2}(0, \nu)=\sin \nu$. Let $\vartheta(\theta, \nu)$ be a continuous branch of the argument of $Y(\theta, \nu)$. The rotation function $\phi(\nu)$ is defined by

$$
\phi(\nu)=\vartheta(2 \pi, \nu)-\vartheta(0, \nu)=\int_{0}^{2 \pi} \frac{d \vartheta(\theta, \nu)}{d \theta} d \theta .
$$

An expression for the rotation function that does not involve $\vartheta$ can be obtained as follows.

$$
\frac{d \vartheta}{d \theta}=\frac{d}{d \theta} \arctan \left(\frac{y_{2}}{y_{1}}\right)=\frac{1}{y_{1}^{2}+y_{2}^{2}}\left|\begin{array}{ll}
y_{1} & \dot{y}_{1} \\
y_{2} & \dot{y}_{2}
\end{array}\right|
$$

Using the fact that the matrix $A$ is symmetric, $Y$ satisfies (4.31), and that for vectors $u$ and $v$ of $\mathbf{R}^{2}$

$$
\operatorname{det}(u, J v)=u^{T} v,
$$

we have that the rotation function satisfies

$$
\phi(\nu)=\int_{0}^{2 \pi}-\frac{Y^{T}(\theta, \nu) A(\theta) Y(\theta, \nu)}{Y^{T}(\theta, \nu) Y(\theta, \nu)} d \theta .
$$

Let

$$
m=\min _{0 \leq \nu \leq \pi} \phi(\nu) \quad \text { and } \quad M=\max _{0 \leq \nu \leq \pi} \phi(\nu) .
$$

The proof of the following lemma can be found in [YS] vol 2 page 662 .

Lemma 4.2 The system (4.31) is unstable if and only if the extreme values $m$ and $M$ of the rotation function satisfy

$$
m<k \pi<M
$$

for some $k \in \mathbf{Z}$

We have the following proposition

Proposition 4.1 Let $z$ be as in (3.3) with $P(\theta)$ a $2 \pi$-periodic $C^{l}$ function satisfying (3.4) and (3.5) and let $J$ and $H$ be the matrices defined in (3.19). Let

$$
\Sigma=\left\{\lambda_{j}^{ \pm} ; j \in \mathbf{Z}\right\}
$$

be the spectrum of the equation

$$
J X^{\prime}(\theta)=\lambda H(\theta) X(\theta) .
$$


Then for every $\lambda_{j}^{ \pm} \in \Sigma$, the fundamental matrix of $(4.37)_{\lambda_{j}^{ \pm}}$is $2 \pi$-periodic.

Proof. We use Fourier series to approximate $H$ by trigonometric polynomials. Let

$$
H(\theta)=\sum_{j \in \mathbf{Z}} H_{j} \mathrm{e}^{i j \theta}, \quad H_{j}=\frac{1}{2 \pi} \int_{0}^{2 \pi} H(\theta) \mathrm{e}^{-i j \theta} d \theta .
$$

For $m \in \mathbf{Z}^{+}$, let

$$
M_{m}(\theta)=\sum_{-m \leq j \leq m} H_{j} \mathrm{e}^{i j \theta}
$$

Then $M_{m}$ is real analytic and converges uniformly to $H$ as $m \longrightarrow \infty$. Consider the corresponding system

$$
J \dot{X}(\theta)=\lambda M_{m}(\theta) X(\theta) .
$$

Let $\phi_{\lambda}(\nu)$ and $\phi_{\lambda}^{m}(\nu)$ be the rotation functions of systems $(4.37)_{\lambda}$ and $(4.40)_{\lambda}^{m}$, respectively. It follows from formula (4.33) and from the definition of $M_{m}$ that $\phi_{\lambda}^{m}(\nu)$ converges uniformly to $\phi_{\lambda}(\nu)$ as $m \longrightarrow \infty$.

Now we complete the proof of the proposition by contradiction. Suppose that there exists $j \in \mathbf{Z}$ such that $\lambda_{j}^{-} \neq \lambda_{j}^{+}$. Then for every $\lambda \in \mathbf{R}$,

$$
\lambda_{j}^{-}<\lambda<\lambda_{j}^{+},
$$

equation $(4.37)_{\lambda}$ is unstable (see remark 3.1). It follows (lemma 4.2) that

$$
\min _{0 \leq \nu \leq \pi} \phi_{\lambda}(\nu)<k \pi<\max _{0 \leq \nu \leq \pi} \phi_{\lambda}(\nu)
$$

for some $k \in \mathbf{Z}$. Consequently, for $m$ large enough we also have

$$
\min _{0 \leq \nu \leq \pi} \phi_{\lambda}^{m}(\nu)<k \pi<\max _{0 \leq \nu \leq \pi} \phi_{\lambda}^{m}(\nu) .
$$

This would mean that the analytic system $(4.40)_{\lambda}^{m}$ is unstable and this is a contradiction (Corollary 4.1)

\section{Structure of the space of infinitesimal bendings.}

For surfaces given as the graph of $z=r^{m} P(\theta)$ with $P>0$ and with positive curvature everywhere except at 0 , we give a complete description of the space of infinitesimal bendings. We use a Fourier method approach to establish our result. 
Let $S$ be the graph of the function $z$ given in polar coordinates by

$$
z(r, \theta)=r^{m} P(\theta)
$$

with $P \in C^{l}, 2 \pi$-periodic, and satisfying

$$
m^{2} P(\theta)^{2}+m P(\theta) P^{\prime \prime}(\theta)-(m-1) P^{\prime}(\theta)^{2}>0 .
$$

Thus, $S$ has positive curvature everywhere except at the flat point 0 . Let $J$ and $H$ be the matrices given by (3.19). It follows from (5.2) that the eigenvalues $h_{-}(\theta)$ and $h_{+}(\theta)$ of $H(\theta)$ are positive for every $\theta$. The spectrum of the equation

$$
J X^{\prime}(\theta)=\lambda H(\theta) X(\theta)
$$

consists of the set $\Sigma=\left\{\lambda_{j}: j \in \mathbf{Z}\right\}$ with $\lim _{j \rightarrow \pm \infty} \lambda_{j}= \pm \infty$ (Proposition 3.1). Furthermore for each $j \in \mathbf{Z}$, the fundamental matrix of $(5.3)_{\lambda_{j}}$ is $2 \pi$-periodic (Proposition 4.1).

Let $L^{2}\left(S^{1} ; \mathbf{R}^{2}\right)$ be the space of square integrable functions from the circle $S^{1}$ to $\mathbf{R}^{2}$. That is

$$
f(\theta)=\left(\begin{array}{c}
f_{1}(\theta) \\
f_{2}(\theta)
\end{array}\right) \in L^{2}\left(S^{1} ; \mathbf{R}^{2}\right) \Longleftrightarrow \int_{0}^{2 \pi} f_{k}^{2}(\theta) d \theta<\infty \text { for } k=1,2 .
$$

Define an inner product in $L^{2}\left(S^{1} ; \mathbf{R}^{2}\right)$ by

$$
(f, g)_{H}=\frac{1}{2 \pi} \int_{0}^{2 \pi} f^{T}(\theta) H(\theta) g(\theta) d \theta
$$

where $f^{T}$ denotes the transpose of $f$. The following proposition establishes the orthogonality of the eigenfunctions of $(5.3)_{\lambda}$ with respect to the inner product $(,)_{H}$.

Proposition 5.1 Let $X_{j}(\theta)$ and $X_{k}(\theta)$ be two eigenfunctions of $(5.3)_{\lambda}$ corresponding to distinct eigenvalues $\lambda_{j}, \lambda_{k} \in \Sigma$. Then

$$
\left(X_{j}, X_{k}\right)_{H}=0 \text {. }
$$

Proof. Suppose that $\lambda_{k} \neq 0$. We have

$$
\begin{aligned}
\left(X_{j}, X_{k}\right)_{H} & =\frac{1}{2 \pi} \int_{0}^{2 \pi} X_{j}^{T} H X_{k} d \theta=\frac{1}{2 \pi \lambda_{k}} \int_{0}^{2 \pi} X_{j}^{T} J X_{k}^{\prime} d \theta \\
& =\frac{1}{2 \pi \lambda_{k}} \int_{0}^{2 \pi}\left(\left(X_{j}^{T} J X_{k}\right)^{\prime}-X_{j}^{\prime} J X_{k}\right) d \theta=\frac{1}{2 \pi \lambda_{k}} \int_{0}^{2 \pi}\left(J X_{j}^{\prime}\right)^{T} X_{k} d \theta \\
& =\frac{\lambda_{j}}{2 \pi \lambda_{k}} \int_{0}^{2 \pi} X_{j}^{T} H X_{k} d \theta=\frac{\lambda_{j}}{\lambda_{k}}\left(X_{j}, X_{k}\right)_{H} .
\end{aligned}
$$


Therefore $\left(X_{j}, X_{k}\right)_{H}=0$ since $\lambda_{j} \neq \lambda_{k}$.

For each $j \in \mathbf{Z}$, let $\left(\phi_{j}(\theta), \psi_{j}(\theta)\right)$ be an orthonormal fundamental matrix of $(5.3)_{\lambda_{j}}$. That is $\phi_{j}$ and $\psi_{j}$ are $2 \pi$-periodic solutions (of class $C^{l-1}$ ) such that

$$
\left(\phi_{j}, \psi_{j}\right)_{H}=0 \quad\left(\phi_{j}, \phi_{j}\right)_{H}=\left(\psi_{j}, \psi_{j}\right)_{H}=1
$$

Proposition 5.2 The system $\left\{\phi_{j}, \psi_{j}\right\}_{j \in \mathbf{Z}}$ forms a basis of $L^{2}\left(S^{1} ; \mathbf{R}^{2}\right)$.

Proof. To prove the completeness of $\left\{\phi_{j}, \psi_{j}\right\}_{j \in \mathbf{Z}}$, we need the asymptotic behavior of the eigenvalues $\lambda_{j}$ and of the fundamental matrix $\left(\phi_{j}, \psi_{j}\right)$. It can be shown (see [YS] Chapter VIII page 776) that the $\lambda_{j}$ 's have the form

$$
\lambda_{j}=\frac{2 \pi}{b_{1}} j+\frac{b_{2}}{b_{1}}+O\left(\frac{1}{|j|}\right)
$$

with

$$
b_{1}=\int_{0}^{2 \pi} \sqrt{\operatorname{det} H(\theta)} d \theta, \quad \text { and } \quad b_{2}=\int_{0}^{2 \pi} \frac{\beta(\theta)}{4 \sqrt{\operatorname{det} H(\theta)}}\left(\ln \frac{\alpha(\theta)}{\gamma(\theta)}\right)^{\prime} d \theta
$$

where we have set

$$
H(\theta)=\left(\begin{array}{cc}
\alpha(\theta) & \beta(\theta) \\
\beta(\theta) & \gamma(\theta)
\end{array}\right) ;\left\{\begin{array}{l}
\alpha(\theta)=\frac{z_{y y}}{m(m-1) r^{m-2} P(\theta)} \\
\beta(\theta)=\frac{-z_{x y}}{m(m-1) r_{x x}^{m-2} P(\theta)} \\
\gamma(\theta)=\frac{z_{x x}}{m(m-1) r^{m-2} P(\theta)}
\end{array}\right.
$$

and $z_{x x}, z_{x y}$, and $z_{y y}$ are given by (3.6).

To obtain the asymptotic behavior of the fundamental matrix, we use the fact that $H(\theta)$ is positive and symmetric to write it as

$$
H(\theta)=\Lambda(\theta)^{T} D(\theta) \Lambda(\theta)
$$

with $\Lambda(\theta)$ orthogonal, $D(\theta)$ diagonal. Let

$$
\Lambda(\theta)=\left(\begin{array}{cc}
\cos k(\theta) & -\sin k(\theta) \\
\sin k(\theta) & \cos k(\theta)
\end{array}\right) \quad \text { and } \quad D(\theta)=\left(\begin{array}{cc}
h_{+}(\theta) & 0 \\
0 & h_{-}(\theta)
\end{array}\right)
$$

where $k(\theta+2 \pi)=k(\theta) \bmod (2 \pi)$ and $0<h_{-} \leq h_{+}$are the eigenvalues of $H$. We can also assume that $h_{-}(0)=h_{+}(0)=1$. Let

$$
Y(\theta)=\Lambda(\theta) X(\theta) \text {. }
$$


It follows from (5.11) and from $\Lambda J \Lambda^{T}=J$ that if $X$ solves (5.3), then $Y$ solves the equation

$$
J Y^{\prime}(\theta)=\left(\lambda D(\theta)+k^{\prime}(\theta) I\right) Y(\theta) .
$$

Define functions $b$ and $\vartheta$ and matrices $R, B_{1}$ and $B_{2}$ as follows

$$
\begin{aligned}
& b(\theta)=\sqrt{\operatorname{det}(D(\theta))}=\sqrt{h_{-}(\theta) h_{+}(\theta)}, \quad \vartheta(\theta)=\frac{i}{2 b(\theta)} k^{\prime}(\theta)\left(h_{+}(\theta)-h_{-}(\theta)\right), \\
& R(\theta)=\left(\begin{array}{cc}
-i b(\theta) & h_{-}(\theta) \\
-h_{+}(\theta) & i b(\theta)
\end{array}\right), \quad B_{1}(\theta)=\left(\begin{array}{cc}
i b(\theta) & 0 \\
0 & -i b(\theta)
\end{array}\right),
\end{aligned}
$$

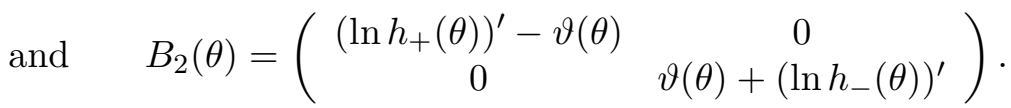

Let

$$
Y_{\lambda}^{0}(\theta)=R(\theta)\left[\exp \int_{0}^{\theta}\left(\lambda B_{1}(s)+B_{2}(s)\right) d s\right] R(0)^{-1}
$$

It can be proved (see [YS] Chapter VIII page 774 ) that as $|\lambda| \rightarrow \infty$, the fundamental matrix $Y_{\lambda}$ of (5.14) satisfies

$$
Y_{\lambda}(\theta)=Y_{\lambda}^{0}(\theta)+O\left(\frac{1}{|\lambda|}\right)
$$

Now, let

$$
c(\theta)=\int_{0}^{\theta} b(s) d s \quad \text { and } \quad l(\theta)=\int_{0}^{\theta} \frac{k^{\prime}(s)\left(h_{+}(s)-h_{-}(s)\right)}{2 b(s)} d s
$$

and let $W_{\lambda}(\theta)$ be the real part of the matrix $Y_{\lambda}^{0}(\theta)$. A direct calculation shows that

$$
W_{\lambda}=\frac{1}{2}\left(\begin{array}{cc}
\left(b h_{+}+h_{-}^{2}\right) \cos (\lambda c-l) & -\left(b h_{+}+h_{-}^{2}\right) \sin (\lambda c-l) \\
\left(b h_{-}+h_{+}^{2}\right) \sin (\lambda c-l) & \left(b h_{-}+h_{+}^{2}\right) \cos (\lambda c-l)
\end{array}\right)
$$

which can be rewritten as

$$
W_{\lambda}=\frac{1}{2}\left(\begin{array}{cc}
b h_{+}+h_{-}^{2} & 0 \\
0 & b h_{-}+h_{+}^{2}
\end{array}\right)\left(\begin{array}{cc}
\cos l & \sin l \\
-\sin l & \cos l
\end{array}\right)\left(\begin{array}{cc}
\cos \lambda c & -\sin \lambda c \\
\sin \lambda c & \cos \lambda c
\end{array}\right) .
$$

The system of $\mathbf{R}^{2}$ valued functions $\left\{E_{j}^{1}, E_{j}^{2}\right\}_{j \in \mathbf{Z}}$ given by

$$
E_{j}^{1}=\left(\begin{array}{c}
\cos \frac{2 \pi j+b_{2}}{b_{1}} c(\theta) \\
\sin \frac{2 \pi j+b_{2}}{b_{1}} c(\theta)
\end{array}\right) \quad E_{j}^{2}=\left(\begin{array}{c}
-\sin \frac{2 \pi j+b_{2}}{b_{1}} c(\theta) \\
\cos \frac{2 \pi j+b_{2}}{b_{1}} c(\theta)
\end{array}\right)
$$


is complete in $L^{2}\left(S^{1} ; \mathbf{R}^{2}\right)$ ( since $c^{\prime}(\theta)=b(\theta)>0$ ). It follows from the standard theory of eigenfunction expansion (see $[\mathrm{BR}]$ page 337 ) that the following system of functions is also complete

$$
F_{j}^{1}(\theta)=\left(\begin{array}{c}
\cos \lambda_{j} c(\theta) \\
\sin \lambda_{j} c(\theta)
\end{array}\right), \quad F_{j}^{2}(\theta)=\left(\begin{array}{c}
-\sin \lambda_{j} c(\theta) \\
\cos \lambda_{j} c(\theta)
\end{array}\right)
$$

where the $\lambda_{j}$ 's are the eigenvalues given asymptotically by (5.8). Consequently, the columns $\phi_{j}^{0}(\theta)$ and $\psi_{j}^{0}(\theta)$ of $W_{\lambda_{j}}(\theta)$ form a complete system in $L^{2}\left(S^{1} ; \mathbf{R}^{2}\right)$ since

$$
\left(\phi_{j}^{0}, \psi_{j}^{0}\right)=M\left(F_{j}^{1}, F_{j}^{2}\right)
$$

with $\operatorname{det}(M)>0$ (see (5.21)). This in turn implies, thanks to (5.17), that the columns of the matrices $Y_{\lambda_{j}}$ form a complete system. Finally, the columns of the fundamental matrices $X_{\lambda_{j}}$ are

$$
\left(\phi_{\lambda_{j}}, \psi_{\lambda_{j}}\right)=\Lambda^{T} Y_{\lambda_{j}}
$$

where $\Lambda$ is the orthogonal matrix given in (5.12), and they form a basis of $L^{2}\left(S^{1} ; \mathbf{R}^{2}\right)$. This completes the proof of the proposition $\triangle$

It follows from Proposition 5.2 that every $f \in L^{2}\left(S^{1} ; \mathbf{R}^{2}\right)$ can be decomposed as

$$
f(\theta)=\sum_{j \in \mathbf{Z}} a_{j} \phi_{j}(\theta)+b_{j} \psi_{j}(\theta)
$$

where

$$
a_{j}=\left(f, \phi_{j}\right)_{H} \quad \text { and } \quad b_{j}=\left(f, \psi_{j}\right)_{H} .
$$

Consider the operator

$$
d_{H}: C^{1}\left(S^{1} ; \mathbf{R}^{2}\right) \longrightarrow L^{2}\left(S^{1} ; \mathbf{R}^{2}\right) \quad d_{H} f(\theta)=H^{-1}(\theta) J f^{\prime}(\theta),
$$

where $f^{\prime}$ is the derivative of $f$. We have then

$$
d_{H} \phi_{j}=\lambda_{j} \phi_{j} \quad \text { and } \quad d_{H} \psi_{j}=\lambda_{j} \psi_{j}
$$

If $f$ differentiable, then it follows from $(5.26)$ and $(5,29)$ that

$$
d_{H} f=\sum_{j \in \mathbf{Z}} \lambda_{j} a_{j} \phi_{j}+\lambda_{j} b_{j} \psi_{j}
$$

More generally, for $f \in C^{k}\left(S^{1} ; \mathbf{R}^{2}\right)$, we have

$$
d_{H}^{k} f=\sum_{j \in \mathbf{Z}} \lambda_{j}^{k} a_{j} \phi_{j}+\lambda_{j}^{k} b_{j} \psi_{j}
$$


(provided that $k<l-2$ ). Consequently, the coefficients $a_{j}$ and $b_{j}$ decay faster than $|j|^{-k}$ as $|j| \rightarrow \infty$. This follows from the standard theory of Fourier series and the asymptotic behavior of $\lambda_{j}$ given in (5.8).

For each $j \in \mathbf{Z}$, let

$$
\begin{aligned}
& V_{j}^{1}(r, \theta)=\rho^{\lambda_{j}} \phi_{j}(\theta)=r^{\lambda_{j}} P(\theta)^{\lambda_{j} / m} \phi_{j}(\theta) \\
& V_{j}^{2}(r, \theta)=\rho^{\lambda_{j}} \psi_{j}(\theta)=r^{\lambda_{j}} P(\theta)^{\lambda_{j} / m} \psi_{j}(\theta)
\end{aligned}
$$

be independent solutions of (3.14), where $\rho=r P^{1 / m}$. To $V_{j}^{1}$ and $V_{j}^{2}$ correspond two independent fields of infinitesimal bendings $U_{j}^{1}$ and $U_{j}^{2}$ of the surface $S$. These fields are given by

$$
\begin{aligned}
& U_{j}^{1}(r, \theta)=r^{\lambda_{j}-(m-1)}\left(r^{m-1} \alpha_{j}^{1}(\theta), r^{m-1} \beta_{j}^{1}(\theta), \gamma_{j}^{1}(\theta)\right) \\
& U_{j}^{2}(r, \theta)=r^{\lambda_{j}-(m-1)}\left(r^{m-1} \alpha_{j}^{2}(\theta), r^{m-1} \beta_{j}^{2}(\theta), \gamma_{j}^{2}(\theta)\right),
\end{aligned}
$$

where $\alpha_{j}^{k}, \beta_{j}^{k}, \gamma_{j}^{k}$ are of class $C^{l-1}$ and $2 \pi$-periodic $(k=1,2)$. The following theorem describes the infinitesimal bendings of $S$.

Theorem 5.1 Let $S$ be a surface given as the graph of a function $z$ as in (5.1), where the positive function $P$ satisfies (5.2). For $j \in \mathbf{Z}$, let $U_{j}^{1}$ and $U_{j}^{2}$ be the fields of infinitesimal bendings given in (5.33). Then for every field of infinitesimal bending $U$ of class $C^{k}(k \leq l-2)$ of the surface $S$ there exist sequences of real numbers $A_{j}$ and $B_{j}$ satisfying

$$
\lim _{j \rightarrow \infty} A_{j} j^{k}=0, \quad \lim _{j \rightarrow \infty} B_{j} j^{k}=0
$$

such that

$$
U(x, y)=\sum_{j} A_{j} U_{j}^{1}(x, y)+B_{j} U_{j}^{2}(x, y) .
$$

Furthermore, $A_{j}=B_{j}=0$ for each $j \in \mathbf{Z}$ such that $\lambda_{j}<m$.

Proof. Let

$$
U(r, \theta)=(\xi(r, \theta), \eta(r, \theta), \zeta(r, \theta))
$$

be a field of infinitesimal bending of $S$ of class $C^{k}$. Let

$$
f=\xi+z_{x} \zeta, \quad g=\eta+z_{y} \zeta, \quad \text { and } \quad V=\left(\begin{array}{c}
f \\
g
\end{array}\right) .
$$

The function $V(\rho, \theta)$ (again $\left.\rho=r P(\theta)^{1 / m}\right)$ satisfies equation (3.14). It follows from proposition 5.2 that

$$
V(\rho, \theta)=\sum_{j \in \mathbf{Z}} p_{j}(\rho) \phi_{j}(\theta)+q_{j}(\rho) \psi_{j}(\theta)
$$


where

$$
p_{j}(\rho)=\left(V(\rho, \theta), \phi_{j}(\theta)\right)_{H} \quad \text { and } \quad q_{j}(\rho)=\left(V(\rho, \theta), \psi_{j}(\theta)\right)_{H} .
$$

Equation (3.14) and expansion (5.38) imply that the functions $p_{j}(\rho)$ and $q_{j}(\rho)$ satisfy the differential equations

$$
p_{j}^{\prime}(\rho)=\lambda_{j} \rho p_{j}(\rho) \quad \text { and } \quad q_{j}^{\prime}(\rho)=\lambda_{j} \rho q_{j}(\rho) .
$$

Thus, there exist constants $A_{j}, B_{j} \in \mathbf{R}$ such that

$$
p_{j}(\rho)=A_{j} \rho^{\lambda_{j}} \quad \text { and } \quad q_{j}(\rho)=B_{j} \rho^{\lambda_{j}} .
$$

Therefore,

$$
V(\rho, \theta)=\sum_{j \in \mathbf{Z}} A_{j} \rho^{\lambda_{j}} \phi_{j}(\theta)+B_{j} \rho^{\lambda_{j}} \psi_{j}(\theta),
$$

In order for $V$ to be of class $C^{k}$ it is necessary that the coefficients $A_{j}$ and $B_{j}$ decay faster that $j^{-k}$ as $|j| \rightarrow \infty$. This combined with the relationships (5.37) shows that the field $U$ has the desired form (5.35). Moreover, it follows from (5.33) and $U$ defined at 0 that $A_{j}=B_{j}=0$ for $\lambda_{j}<m$. This completes the proof of the theorem $\triangle$

\section{References.}

[A] A. Aleksandrov, Infinitesimal bendings of nonregular surfaces, Mat. Sb., Nov. Ser. 1, 307-322, (1937)

[BG] M. Berger and B. Gostiaux, Differential geometry: manifolds, curves, and surfaces (section 11.14), GMT 115, Springer Verlag, (1988)

[BR] G. Birkhoff and G. C. Rotta, Ordinary differential equations, 2nd ed., Blaisdell Publ. Co., (1969)

[C] S. Cohn-Vossen, Bendability of surfaces in the large, Usp. Mat. Nauk. 1, 33-76, (1936)

[D] G. Darboux, Leçons sur la théorie générale des surfaces, Part 4, Gauthier-Villars, Paris, (1894)

[E1] N. V. Efimov, Qualitative questions of the theory of deformation of surfaces, Usp. Mat. Nauk 3, No 2, 47-128, (1948). Transl. AMS Transl. 6, 274-323 
[E2] N. V. Efimov, On the rigidity in the small, Dokl. Akad. Nauk SSSR 60, 761-764, (1948)

[EU] N. V. Efimov and Z. D. Usmanov, Infinitesimal bendings of surfaces with a flat point, Dokl. Akad. Nauk SSSR 208, 28-31, (1973). Transl. Sov. Math. Dokl. 14, 22-25

[K] E. Kann, A new method for infinitesimal rigidity of surfaces with $K \geq$ 0, J. Diff. Geom. 4, 5-12, (1970)

[M1] A. Meziani, On planar elliptic structures with infinite type degeneracy, J. Funct. Analysis 179, 333-373, (2001)

[M2] A. Meziani Generalized CR equation with a punctual singularity, To appear in Complex Variables.

[P] A. V. Pogorelov, Bendings of surfaces and stability of the shells, Nauka Moscow (1986). Transl. AMS Providence, RI, (1988)

[S1] I. K. Sabitov, Some results on infinitesimal bendings of surfaces in the small and in the large, Dokl. Akad. Nauk SSSR 162, 1256-1258, (1965). Transl. Sov. Math. Dokl. 6, 862-864

[S2] I. K. Sabitov, Investigations of the rigidity and non-bendability of analytic surfaces of revolution with flattening at the pole, Vestn. Univ. Ser. I, 5, 29-36, (1986). Transl. Mosc. Univ. Math. Bull.41, 33-41

[S3] I. K. Sabitov, Local theory of bending of surfaces, Encyclopaedia of Math. Sci., vol 48, Springer-Verlag (1992)

[S] M. Spivak, A comprehensive introduction to differential geometry, vol 5, 2nd ed., Publish or Perish, Berkeley, (1975)

[T] F. Treves, Hypo-analytic structures: local theory, Princeton Univ. Press., (1992)

[U1] Z. D. Usmanov, On Efimov surfaces that are rigid in the small, Mat. Sb. 187 (1996), no 6, 119-130. Transl. in Sb. Math 187, no 6, 903-915

[U2] Z. D. Usmanov, Generalized analytic functions in differential geometry, 127-138, in Generalized Analytic Functions (Graz 1997), Int. Soc. Anal. Appl. Comput., 1, Kluwer Acad. Publ., Dordrecht, (1998)

[V] I. N. Vekua, Generalized analytic functions, Pergamon Press, London (1962) 
[Y] M. Yoshida, Fuchsian differential equations, Aspect of Mathematics, E 11, Friedr. Vieweg, (1987)

[YS] V. A. Yakubovich and V. M. Starzhinskii, Linear differential equations with periodic coefficients, vol 2, John Wiley, (1975)

DEPARTMENT OF MATHEMATICS

FLORIDA INTERNATIONAL UNIVERSITY

MiAmi, FloRIDA 33199

meziani@fiu.edu

ReCEIVED FEBRUARY 4, 2002. 